\title{
Magnetic Tunnel Junction Magnetic Field Sensor Design Tool
}

\author{
Ronald C. L. Li ${ }^{1}$, J. Unguris ${ }^{2}$, Alan S. Edelstein ${ }^{3}$, J. E. Burnette ${ }^{3}$, G. A. Fischer ${ }^{3}$, Edmund R. Nowak ${ }^{4}$, William F. \\ Egelhoff, Jr. ${ }^{5}$, and Philip W. T. Pong ${ }^{1 *}$, Member, IEEE
}

\begin{abstract}
A spreadsheet-based magnetic tunnel junction (MTJ) sensor design tool is presented in this paper. The system is developed using Excel and Visual Basic Application. It allows users to optimize the various parameters of the sensor design with the goal of SQUID-like sensitivity. Users can input parameters of the design including magnetic properties, junction areas, and free layers thicknesses. The design tool will then calculate and display automatically various noise sources including Johnson noise, shot noise, 1/f noise, and thermal magnetic noise that must be considered when building MTJ magnetic field sensors. Graphs predicting the sensitivities, operating current and power of the finished sensors are shown and fine tuning of each design parameter is allowed using the scrollbars provided. Using this design tool, effects of changes made to any design parameter can be clearly observed and detailed noise analysis can be studied without manually repeating complex calculations.
\end{abstract}

\section{INTRODUCTION}

To design a ultrasensitive magnetic tunnel junction (MTJ) magnetic field sensor with sensitivity down to 1 picoTesla, calculations needs to be performed iteratively to plot noise analysis graphs for analyzing the MTJ sensor performance [13]. Moreover, there are 15 design parameters needed to be studied and their effects on sensor performance are intertwined. The complexity and the time needed for a complete noise analysis make the design optimization task impossible to be handled manually. An automatic calculation system that enables scientists to observe the influence of each design parameter on sensor performance in real-time will be greatly conducive to the development of MTJ sensors.

\section{MTJ MAGNETIC SENSOR DESIGN TOOL}

A spreadsheet-based MTJ magnetic sensor design tool is presented in this paper. It can re-calculate the entire sheet automatically whenever an update to a single cell is made [4]. The system was developed using Microsoft Excel and Visual Basic Application (VBA). Eq. (1) and (2), based on our mathematical derivations [5], show the calculation of the total

${ }^{1}$ Department of Electrical and Electronic Engineering, University of Hong Kong, Pokfulam Road, Hong Kong, *Email: ppong@eee.hku.hk

${ }^{2}$ Center for Nanoscale Science and Technology, National Institute of Standards and Technology (NIST), Gaithersburg, Maryland MD 20899, USA

${ }^{3}$ Army Research Laboratory, Adelphi, Maryland MD 20783, USA

${ }^{4}$ Department of Physics and Astronomy, University of Delaware, Newark, Delaware 19716, USA

${ }^{5}$ Metallurgy Division, National Institute of Standards and Technology (NIST), Gaithersburg, Maryland MD 20899, USA noise composed of amplifier noise, shot and Johnson noise, electronic $1 / \mathrm{f}$ noise, thermal magnetic noise, and magnetic $1 / \mathrm{f}$ noise.

$$
\begin{aligned}
& S_{B}=S_{B}^{A m p}+S_{B}^{\text {shot }}+S_{B}^{\text {elec.1/f }}+S_{B}^{\text {therm..mag }}+S_{B}^{\text {mag.1/ } f} \\
& S_{B}=\frac{4 B_{\text {sat }}^{2}}{(\Delta R / \bar{R})^{2} N^{2} V_{J}^{2}}\left[S_{V}^{A m p}+N \frac{2 e V_{J}[R A P]}{A} \operatorname{coth}\left(\frac{e V_{J}}{2 k_{B} T}\right)+N \frac{\alpha_{\text {elect }} V_{J}^{2}}{A f}\right] \\
& +\frac{1}{N} \frac{4 k_{B} T \mu_{0} \alpha_{G}}{\Omega \gamma M_{s}}+\frac{2 B_{\text {sat }}}{N} \frac{\alpha_{\text {mag }}}{\Omega f}
\end{aligned}
$$

In eq. (2), $\alpha_{\text {mag }}$ is magnetic $1 / \mathrm{f}$ noise Hooge parameter, $\mathrm{A}$ is MTJ area, [RAP] is resistance-area product, $\mathrm{f}$ is detection frequency, $\alpha_{\text {elect }}$. is electronic Hooge parameter, $\Omega$ is free-layer volume, $\alpha_{\mathrm{G}}$ is Gilbert damping parameter, $V_{J}$ is junction voltage, and $\mathrm{N}$ is number of MTJs per leg. The sensor model in this design tool is a MTJ sensor integrated with a MEMS flux concentrator which is used to eliminate $1 /$ f noise [6]. Users can input sensor design parameters including junction voltage, number of MTJs in each leg of the Wheatstone bridge, junction area, saturation field, tunneling magnetoresistance (TMR), flux concentrator gain, amplifier noise, operating temperature, junction resistance-area product (RA), magnetic free layer saturation magnetization, damping alpha, electronic $1 / \mathrm{f}$ noise alpha, operating frequency, free layer thickness, and magnetic $1 / \mathrm{f}$ noise alpha. The effects on various kinds of MTJ sensor noises are calculated by eq. (1) and (2) and visualized immediately through graphical displays. Fine tuning on each design parameter is enabled using the scrollbars provided in the Tool Menu and the noise analysis is displayed in real-time.

\section{RESULTS AND DISCUSSION}

This Excel spreadsheet design tool is divided into the (i) design mode (fig. 1) and (ii) implement mode (fig. 2). In the design mode, modifications of equations and input of various data can be carried out and the sensor performance calculation results are shown in numerical forms. In the implement mode, performance of the sensor defined in the design mode is shown in graphical forms. By using this design tool, a possible sensor design with sensitivity close to 1 picoTesla per sqrt $\mathrm{Hz}$ is obtained. 


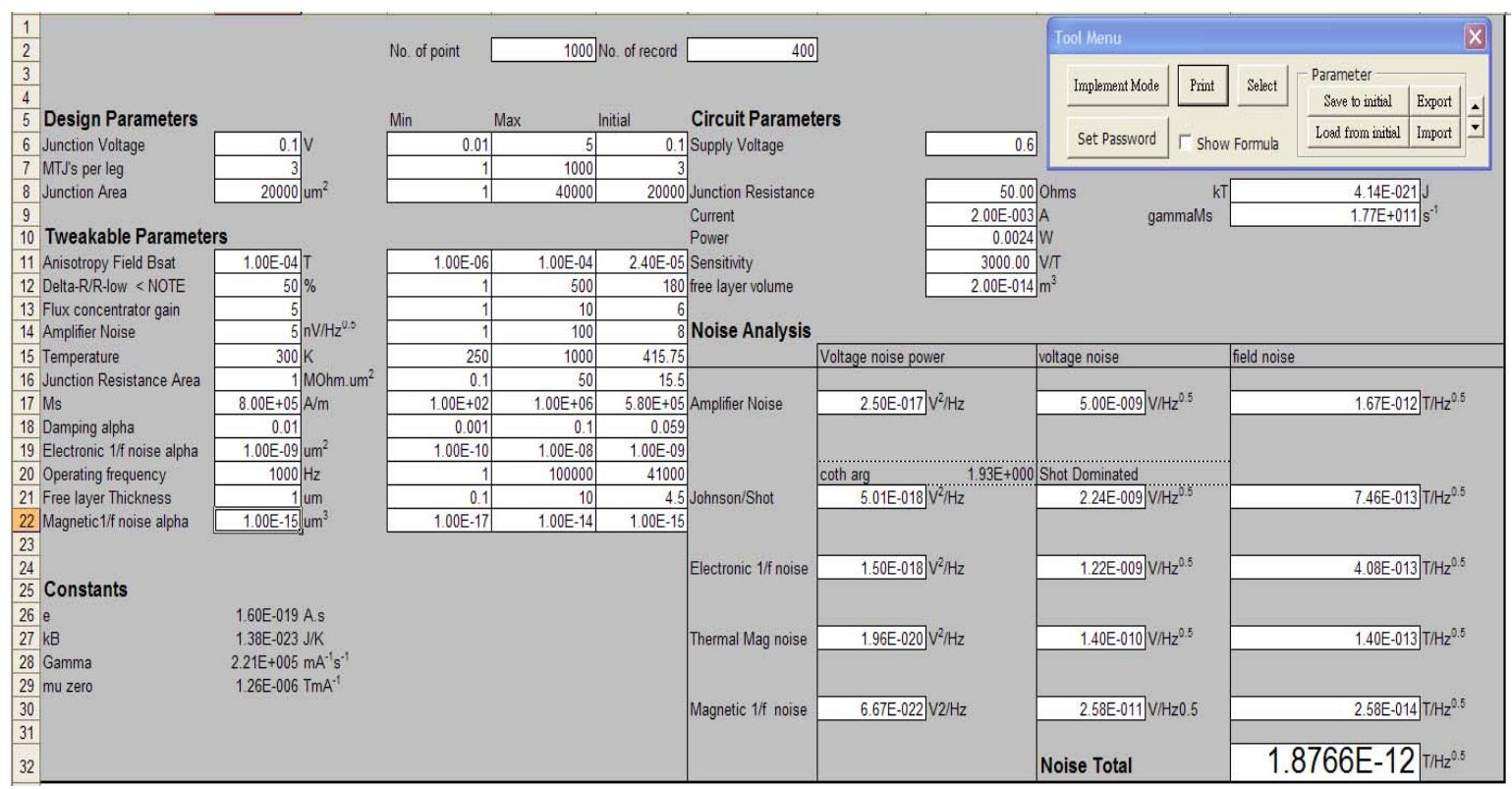

Fig. 1. Interface of the design mode with Tool Menu. This is for users inputting design parameters (left hand side of the interface) and displaying sensor performance calculation results in numerical forms (right hand side) in real-time. The Tool Menu (top right corner) enables switching between implement mode and design mode, exporting and importing design configurations, saving graph data, and printing the results.

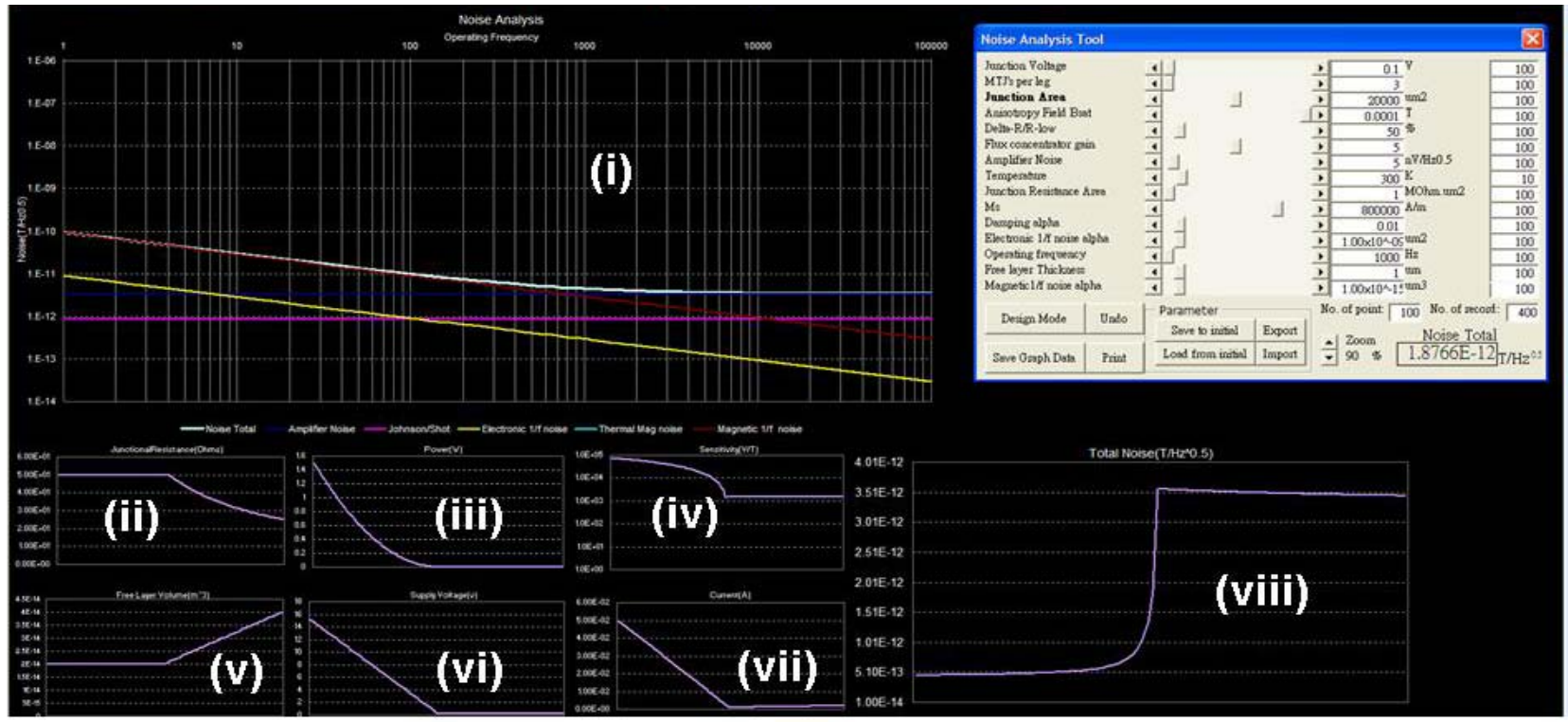

Fig. 2. Interface of the implement mode with (i) Noise Analysis Graph (ii) Junction Resistance Graph (iii) Power Graph (iv) Sensitivity Graph (v) Free Layer Volume Graph (vi) Supply Voltage Graph (vii) Current Graph (viii) Total Noise Graph and Tool Menu at the top right corner. Graph (i) displays the amplifier noise, shot and Johnson noise, electronic 1/f noise, magnetic $1 / \mathrm{f}$ noise, and thermal magnetic noise. The graphs (ii) to (vii) and graph (viii) show the present and previous values of the design parameters and the total noise level to illustrate the effects of design parameter changes on total noise level.

\section{References}

[1] M. Tondra, J. M. Daughton, D. Wang, R. S. Beech, A. Fink, and J. A. Taylor, J. Appl. Phys. 83, 6688 (1998)

[2] N. A. Stutzke, S. E. Russek, and D. P. Pappas, J. Appl. Phys. 97, 10Q107 (2005)

[3] C. Ren, X. Liu, B. D. Schrag, and G. Xiao, Phys. Rev. B 69, 104405 (2004)
[4] Robert de Levie (2004). Advanced Excel for Scientific Data Analysis. New York: Oxford.

[5] W. F. Egelhoff, Jr., P. W. T. Pong, J. Unguris, R. D. McMichael, E. R. Nowak, A. S. Edelstein, J. E. Burnette, and G. A. Fischer, Sens. Actuators A: Phys. (2009), doi:10.1016/j.sna.2009.08.016

[6] J. E. Burnette, A. S. Edelstein, G. A. Fischer, E. Nowak, W. Bernard, S. F. Cheng, C. Nordman, and W. F. Egelhoff, Jr., J. Appl. Phys. 103, 07E930 (2008) 